\title{
Facet temperature reduction by separate pumped window in high power laser diodes
}

Seval Arslan, Sinan Gündoğdu, Abdullah Demir, Atilla Aydınlı

Seval Arslan, Sinan Gündoğdu, Abdullah Demir, Atilla Aydınlı, "Facet temperature reduction by separate pumped window in high power laser diodes," Proc. SPIE 10682, Semiconductor Lasers and Laser Dynamics VIII, 106822B (9 May 2018); doi: 10.1117/12.2311642

SPIE. Event: SPIE Photonics Europe, 2018, Strasbourg, France 


\title{
Facet temperature reduction by separate pumped window in high power laser diodes
}

\author{
Seval Arslan*a, Sinan Gündoğdu ${ }^{\mathrm{a}}$, Abdullah Demir ${ }^{\mathrm{a}}$, Atilla Aydınlı ${ }^{\mathrm{b}}$ \\ ${ }^{a}$ Dept. of Physics, I.D. Bilkent Univ., Ankara, TURKEY 06800; \\ ${ }^{\mathrm{b}}$ Dept. of Electrical and Electronics Engineering, Uludağ Univ., Bursa, TURKEY 16059
}

\begin{abstract}
The main optical output power limitation in high power laser diodes is the catastrophic optical mirror damage (COMD) initiated by facet heating due to optical absorption, which limits the reliable power and lifetime of a single laser. Facet heating correlated with current injection near laser facets can be reduced by unpumped window structure. However, the high-power laser slope efficiency drops as the length of the window increases. In this work, separately pumped window (SPW) method is proposed and experimentally demonstrated to significantly reduce the facet temperature of the semiconductor lasers without compromising their performance. We used 5-mm long high-power laser diodes and compared its performance and facet temperature to the devices integrated with SPW facet sections, which are electrically isolated from the laser section. The slope efficiencies of the lasers with SPW and that of 5-mm lasers without SPW are comparable when SPW is pumped at its transparency current, illustrating that SPW integrated lasers preserve their slope efficiency. As the window pumping current increases, the threshold current of the laser with SPW decreases when the SPW approaches transparency. The facet temperature rise $(\Delta \mathrm{T})$ of the lasers were measured by the thermoreflectance method. The $\Delta \mathrm{T}$ measured at waveguide regions of lasers was shown to be reduced by $42 \%$ implementing SPW region to conventional lasers. Therefore, SPW technique was shown to be a promising approach to increase the COMD level of the high-power laser diodes and it opens up a new avenue for reliable semiconductor laser operation at very high output power levels.
\end{abstract}

Keywords: Facet temperature, thermoreflectance, high power laser diodes, catastrophic optical mirror damage.

\section{INTRODUCTION}

Semiconductor lasers attract much attention due to the high output power and conversion efficiency ${ }^{1}$. However, catastrophic optical mirror damage limits reaching higher power levels mainly due to facet heating initiated by optical absorption at the facet ${ }^{2}$. Facet absorption becomes more prominent at high temperatures and results in catastrophic device failure ${ }^{3}$. Many approaches were found to prevent heat load at high power laser facets with different packaging methods. To circumvent this problem, high-power laser diodes are mounted epi-down for effective heat spreading using different solders, for example, AuSn solders that lead to better thermal resistances ${ }^{4-6}$. Alternatively, process-based solutions are investigated to improve the reliable power limits are employed such as non-absorbing mirrors (NAMs) and inclusion of an unpumped region at close vicinity of the laser facets. NAMs aim to increase bandgap near the laser facets to prevent optical absorption ${ }^{7-9}$. Unpumped windows keep hot region away from the laser facets. However, the length of the unpumped region is restricted by optical reabsorption that leads to reduce of quantum efficiency of high power lasers $^{10}$

In this work, facet temperature is reduced using a SPW region near the front facet of the InGaAs/AlGaAs high power laser diodes for conservation of quantum efficiency. Lasers are separated into two electrically isolated electrodes. The longer one is pumped for lasing. The other region near to front facet is pumped at transparency to prevent optical absorption while keeping facet temperature lower and transparency current is very low to generate extra heat at window. Facet temperature measurements and characteristic laser performance measurements were done to demonstrate the effective facet temperature reduction. Micro-Raman spectroscopy and thermoreflectance are the highly preferred techniques to measure facet temperature ${ }^{7,8,11-16}$. However, here thermoreflectance method was used due to its high resolution below $1 \mathrm{~K}^{14,17}$. There are two approaches to extract the temperature map of the laser facet; one is the point by point mapping using a single photodetector and the other is direct imaging of the facet by camera-based thermoreflectance method that is used in this work. The facet temperature rises of the 5-mm conventional high-power

Semiconductor Lasers and Laser Dynamics VIII, edited by Krassimir Panajotov, Marc Sciamanna, Rainer Michalzik, Proc. of SPIE Vol. 10682, 106822B · (c) 2018 SPIE · CCC code: 0277-786X/18/\$18 · doi: 10.1117/12.2311642 
laser was compared with that of the laser with a 5-mm long laser with a 1-mm long SPW region. Measured facet temperature rise was reduced by up to $42 \%$ using this approach. The slope efficiency of the laser with SPW section was observed to increase above that of the conventional laser. Our results demonstrate that the facet temperatures can be manipulated with this approach even at high output powers and make it possible to increase COMD threshold level.

\section{EXPERIMENTAL}

The epitaxial structure of the laser used in this work is the same as that used in previously ${ }^{18}$. In this work, the facet temperatures of the conventional lasers with short section of unpumped windows is compared with that of the laser including electrically isolated SPW region close to the front facet in addition to the small length of unpumped window. We, also demonstrate that laser slope efficiency is not sacrificed at operating currents. The cross-sectional schematics from the contact regions of the conventional laser and that of the laser with SPW region are shown in Fig. 1. We followed the same process steps as conventional lasers to fabricate them. A $100 \mu \mathrm{m}$ waveguide regions were defined by wet etching, $\mathrm{Si}_{3} \mathrm{~N}_{4}$ deposited on sample by plasma enhanced chemical vapor deposition (PECVD) as the passivation layer. $\mathrm{Si}_{3} \mathrm{~N}_{4}$ was removed from the p-metal contact regions of the ridge waveguides and followed by the deposition top and bottom contact metals. Alloyed was formed by RTA process. For the lasers with SPW, electrical isolation between two distinct regions was achieved by $300 \mathrm{~nm}$ deep trench. Finally, lasers were cleaved and mounted epi-up on copper heat sink.

All the lasers were analyzed under continuous wave $(\mathrm{CW})$ operation mode up to the $3 \mathrm{~A}$ operation current at room temperature. The slope efficiencies of the conventional lasers were compared with that of the lasers with SPW for various window currents $\left(\mathrm{I}_{\mathrm{w}}\right)$ of $0,50,100,150$ and $180 \mathrm{~mA}$.

$\Delta \mathrm{T}$ was determined by camera-based thermoreflectance method using "four bucket" technique ${ }^{10}$. Signal to noise ratio was increased by software based averaging. Fig.2 illustrates the temperature measurement set-up. Laser facets was illuminated by a probe light LED at $450 \mathrm{~nm}$ through a microscope objective with 20x magnification and 0.4 NA and reflected light was analyzed by camera triggered by a diode driver. Reflectivity changes with surface temperature is expressed as ${ }^{11,15,19,20}$

$$
\frac{\Delta R}{R}=\left(\frac{1}{R} \frac{\partial R}{\partial T}\right) \Delta T=\kappa \Delta T
$$

where $\kappa$ is the thermoreflectance coefficient and related to the material and wavelength of the probe light. We found the thermoreflectance coefficient of GaAs as $3.2 \pm 0.6 \times 10^{-4}$ by modulating laser temperature using Newport 3700 temperature controller. The epitaxial structure of our lasers is composed of AlGaAs layers with different Al content. Therefore, $\kappa$ constant of the epitaxial layers is a little bit different from the GaAs. However, this situation is not critical for us because the aim of this work is to show facet temperature reduction of lasers with SPW with respect to the facet temperature of the conventional lasers. The temperature and spatial resolution is $\pm 0.1{ }^{\circ} \mathrm{C}$ and $\pm 0.4 \mu \mathrm{m}$. 
a)

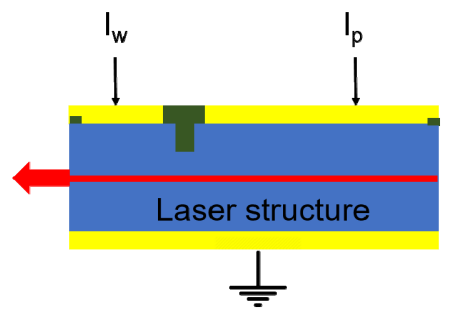

b)

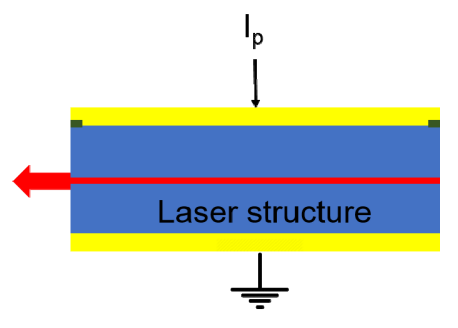

Passivation region

Metal contacts

Active region

Figure 1. a) The schematic illustration of the lasers with SPW region and b) that of the conventional lasers. The crosssectional view from the waveguide region is given this figure.

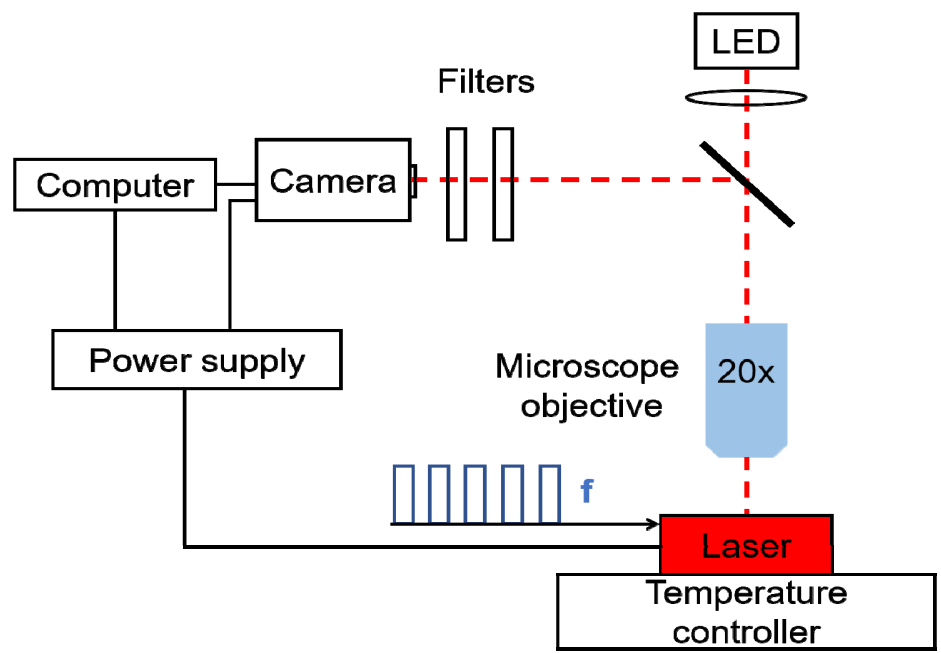

Figure 2. Experimental set-up for camera based-thermoreflectance measurement set-up. A LED at $450 \mathrm{~nm}$ is used as probe light.

\section{RESULTS AND DISCUSSIONS}

In Fig.3a the temperature distribution map of $4.00+1.00 \mathrm{~mm}$ laser at 3.17A injection current is given and in the Fig. $3 \mathrm{~b}$ the $\Delta \mathrm{T}$ line scan of 5.00-mm long conventional laser at $3 \mathrm{~A}$ and that of $5-\mathrm{mm}$ long laser with SPW region at 3.17A from top surface to the GaAs substrate is represented. Sharp changes in line scans are due to interface effects and the material changes of the epitaxial layer. Epitaxial layers are composed of very thin InGaAs, and relatively thick AlGaAs layers with different Al contents. Therefore, the calibration coefficient of epitaxial layers differs slightly from the GaAs. We calculated the temperature rise, $\Delta \mathrm{T}$, for 5-mm long laser without the SPW and the laser with SPW assuming the calibration coefficient of all layers are equal to that of GaAs. Temperature errors caused by differences in calibration coefficients of different layers is not critical because our aim is to report facet temperature changes for 5-mm long laser with 1-mm long SPW region with the same epitaxial structure. We compare the temperature changes, $\Delta \mathrm{T}$, of these two types of lasers in the active region including waveguide layers with a spatial resolution of $\pm 400 \mathrm{~nm}$. The temperature rises for the 5-mm laser with and without SPW region are equal to 19 and $34{ }^{\circ} \mathrm{C}$ at 3.17 and $3.00 \mathrm{~A}$, respectively. The temperature rise between the lasers was reduced by $42 \%$ when the SPW section is at transparency. 
(a)

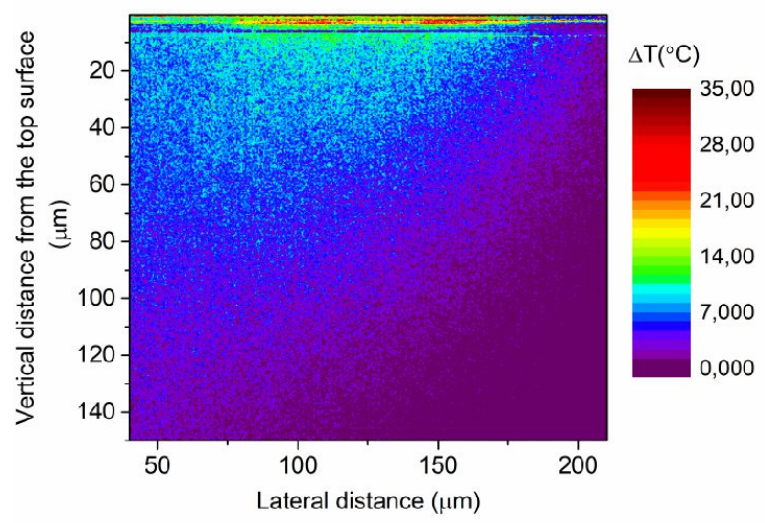

(b)

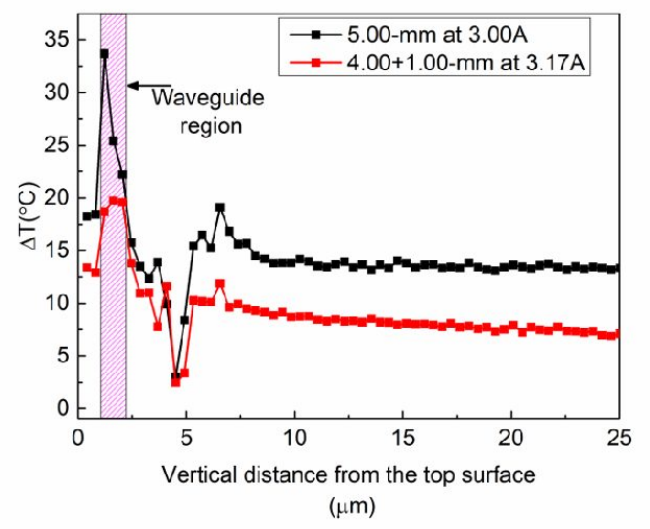

Figure 3. (a) Temperature map of $4.00+1.00 \mathrm{~mm}$ long laser at 3.17A. (b) Temperature line scans for 5.00-mm long conventional laser and $5.00-\mathrm{mm}$ long laser with a $1.00-\mathrm{mm}$ long window region taken at the active region through the direction perpendicular to surface.

In Fig.4 we compare the slope efficiency and threshold currents of the 5-mm long conventional laser with those lasers with 1-mm long SPW. We found the slope efficiencies by taking the twice of output power measured from the front facet which showed a slight increase when compared to the conventional laser. The slope efficiency of the lasers with 1$\mathrm{mm}$ long SPW region may be expected to be slightly larger than that of the 5-mm ordinary laser since the facet temperature of the lasers with SPW at transparency is lower than that of the conventional laser due to smaller window loss. In contrast, slope efficiency of the lasers with SPW decreased by $\sim 40 \%$ when the window region was not biased. On the other hand, threshold currents of the lasers with SPW decreases below the threshold current of conventional lasers with increasing window bias current because optical loss decreases with increasing window bias currents. The slope efficiencies of the $5-\mathrm{mm}$ long conventional laser and 5-mm long laser with 1-mm long SPW region are 0.99 and $1.00 \mathrm{~W} / \mathrm{A}$ at $150 \mathrm{~mA}$ windows bias current, respectively. Beyond window bias current of $170 \mathrm{~mA}$, slope efficiency perceptively increases indicating gain in the SPW section. We, therefore, determine the threshold current of SPW as 170 $\mathrm{mA}$. We note that the threshold current of the $5-\mathrm{mm}$ long conventional laser is equal to $570 \mathrm{~mA}$. We also note that the 5$\mathrm{mm}$ long laser with 1-mm long SPW reaches threshold when the sum of the threshold current and window bias current is $570 \mathrm{~mA}$. 


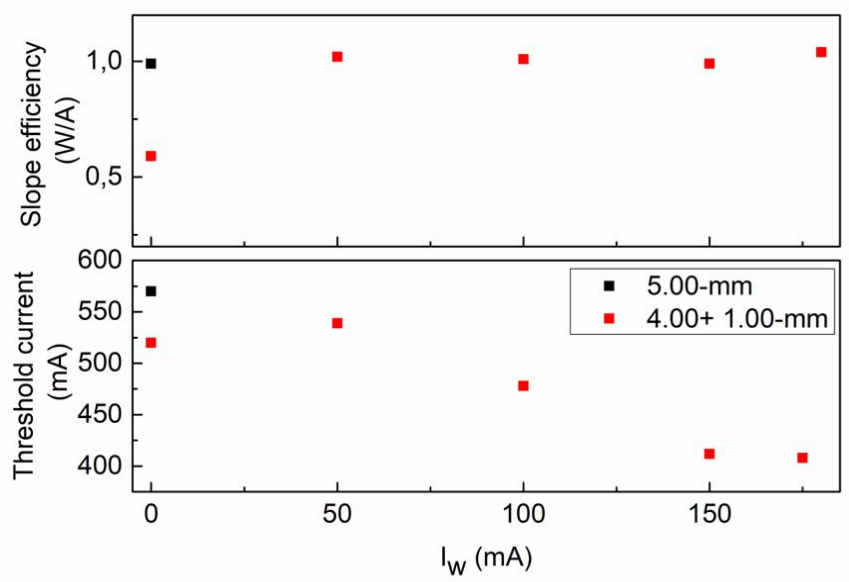

Figure 4. (Top and bottom) Slope efficiency and threshold current of 5-mm long ordinary laser and 5-mm long laser with SPW region for various window currents.

In Fig.5 we compare the temperature rise of 5-mm long conventional laser with 5-mm long laser with 1-mm SPW region at injection current of $3 \mathrm{~A}$ for various window currents. The transparency current can also be determined from the $\Delta \mathrm{T}$ behavior of the 5-mm long laser with SPW section at various window bias currents. When the window bias current of the laser with a SPW section is smaller than the transparency current, the $\Delta \mathrm{T}$ of this laser is larger than the $\Delta \mathrm{T}$ of SPW laser with no bias current. This is due to Joule heating of the bias current. $\Delta \mathrm{T}$ decreases as the transparency is approached, reaching a minimum of $19^{\circ} \mathrm{C}$ at a window bias current of $0.17 \mathrm{~A}$. When we compared this temperature change with $\Delta \mathrm{T}$ of 5 -mm long laser without SPW, we find that $\Delta \mathrm{T}$ is reduced by $42 \%$ with pumped window approach.

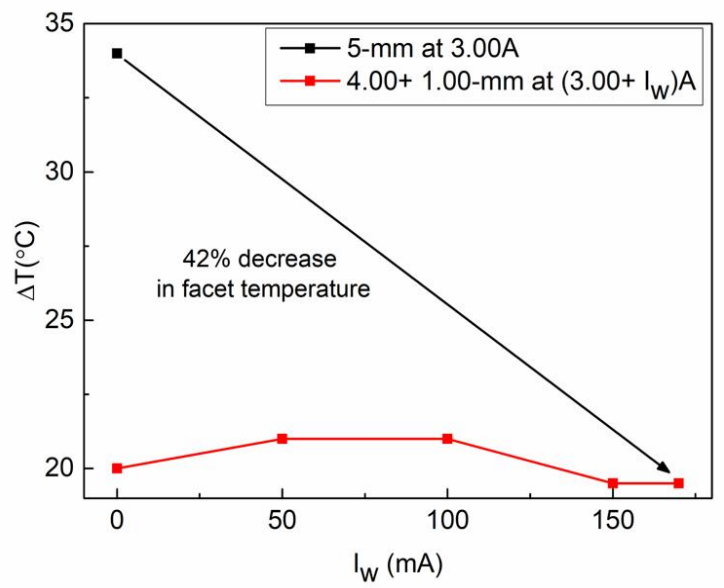

Figure 5. The comparison of the temperature change of 5-mm long laser with 5-mm long laser with 1-mm SPW region for various window currents.

\section{SUMMARY}

In the quest for ever higher optical powers from laser diodes, COMD is still a major obstacle. Despite the improvements in the COMD threshold with various approaches, even higher damage thresholds are still desired. In this report, we introduce a new concept by employing longer unpumped sections for lower facet temperatures that are otherwise biased to transparency to eliminate the optical loss due to length of these sections. We have demonstrated that this concept 
allows facet temperature management by preserving laser performance. The facet temperature rise in 5-mm ordinary lasers was reduced by $42 \%$ at $3 \mathrm{~A}$ employing a $1-\mathrm{mm}$ long SPW region. The facet temperatures can be decreased even to lower temperatures by optimizing the window length with temperature. Slope efficiency of the separately pumped window laser is brought to same level or slightly above when the window region is biased at the transparency current. This new design allows to reach higher reliable output powers than existing power records.

\section{Acknowledgements}

The financial support of Ermaksan A.Ş. is gratefully acknowledged.

\section{REFERENCES}

[1] Zucker, E. et al., "Adavancements in laser diode chip and packaging technologies for application in kW-class fiber laser pumping," Proc. of SPIE 8965, 896507 (2014).

[2] Ziegler, M., Hempel, M., Larsen, H. E., Tomm, J. W., Andersen, P.E., Clausen, S., Elliott, S. E., and Elsaesser, T., "Physical limits of semiconductor laser operation: A time-resolved analysis of catastrophic optical damage," Appl. Phys. Lett., p.97 (2010).

[3] Hempel, M. et al., "catastrophic optical damage at front and rear facets of diode lasers," Appl. Phys. Lett., 97, $231101(2010)$.

[4] Liu, X. et al., "Comparison between epi-down and epi-up bonded high-power single-mode 980-nm semiconductor lasers," IEEE Transactions on Advanced Packaging, 27, 4 (2004).

[5] Hostetler, J. L., "Thermal and strain characteristics of high-power $940 \mathrm{~nm}$ laser arrays mounted with AuSn and In solders," Proc. of SPIE 6456, 645602 (2007).

[6] Teo, J. W. R., Li, G. Y., Ling, M. S., Wang, Z. F., and Shi, X. Q., "Parametric investigation of laser diode bonding using eutectic AuSn solder," Thin Solid Films, 515 (2007).

[7] Piva, P. G., Fafard, S., Dion, M., Buchanan, M., Charbonneau, S., Goldberg, R. D., and Mitchell, I. V., "Reduction of InGaAs/GaAs laser facet temperatures by band gap shifted extended cavities," Appl. Phys. Lett., 70 (1997).

[8] Michaud, J., Vecchio, P. D., Bechou, L., Veyrie, D., Bettiati, M. A., Laruelle, F., and Grauby, S., "Precise facet temperature distribution of high-power laser diodes. Unpumped window effect," IEEE Phot.Techn. Lett., 27 (2015).

[9] Rinner, F., Rogg, J., Kelemen, M. T., Mikulla, M., Weimann, G., Tomm, J. W., Thamm, E., and Poprawe, R., "Facet temperature reduction by a current blocking layer at the front facets of high-power InGaAs/ AlGaAs lasers," Journal of Applied Physics, 93 (2003).

[10] Hendrix, J., Morthier, G., and Baets, R., "Influnce of laser parameters and unpumped regions near the facets on the power level for catastrophic optical damage in short wavelength lasers.” IEEE Proc.-Optoelectronics, 144 (1997).

[11]Farzaneh, M., Maize, K., Lüerben, D., Summers, J. A., Mayer, P. M., Raad, P. E., Pipe, K. P., Shakouri, A., Ram, R. J., and Hudgigngs, J. A., "CCD-based thermoreflectance microscopy: principles and applications," Journal of Physics D: Appl. Phys., 42 (2009).

[12] Pierscinska, D., "Thermoreflectance spectroscopy-Analysis of thermal processes in semiconductor lasers," Journal of Pyhsics D: Appl. Phys., 51 (2018).

[13] Epperlein, P. W., Bona, L. G., and Roentgen, P., "Local mirror temperatures of red-emitting (Al)GaInP quantumwell laser diodes by Raman scattering and reflectance modulation measurements," Appl. Phys. Lett., 60 (1992).

[14] Chan, P. K. L., Pipe, K. P., Plant, J. J., Swint, R. B., and Juodawlkis, P. W., "Temperature mapping and thermal lensing in large-mode, high-power laser diodes," Appl. Phys. Lett., 89 (2006).

[15] Mayer, P. M., Lüerben, D., Ram, R. J.,and Hudgings, J. A., "Theoratical and experimental investigation of the thermal resolution and dynamic range of CCD-based thermoreflectance imaging," Journal of Optical Society of America A, 24 (2007). 
[16] Sanayeh, M. B., Brick, P., Schmid, M., Mayer, B., Müller, M. et al., "The physics of catastrophic optical damage in high-power AlGaInP laser diodes," Proc. of SPIE 6997, 699703 (2008).

[17] Schaub, E., "Optical absorption rate determination, on the front facet of high-power GaAs laser diodes, by means of thermoreflectance technique," Japanese Journal of Applied Physics, 40 (2001).

[18] Arslan, S., Demir, A., Şahin, S., and Aydınlı, A., "Conservation of quantum efficiency in quantum well intermixing by stress engineering with dielectric bilayers," Semiconductor Science and Technology, 33 (2018).

[19] Epperlein, P. W., "Micro-temperature measurements on semiconductor laser mirrors by reflectance modulation: A newly developed technique for laser characterization,” Japanese Journal of Applied Physics, 32 (1993).

[20] Wawer, D., Ochalski, T. J., Pierscinski, K., Szymanski, M., Bugajski, M. et al., "Analysis of facet heating in semiconductor lasers," Proc. of SPIE 7009, 70090C (2005). 